\title{
A Escala de Analogia Visual do Apetite como ferramenta de apoio na detecção do risco de fracasso da persistência de perda de peso de pacientes com obesidade submetidos à gastroplastia
}

\author{
The Appetite Visual Analogy Scale as a support tool in detecting the risk of failure of the \\ persistence of weight loss in patients with obesity undergoing gastroplasty
}

La Escala de Analogía Visual del Apetito como herramienta de apoyo en la detección del riesgo de fracaso de la perdida de peso persistente en pacientes com obesidad sometidos a gastroplastia

\section{Resumo}

Objetivou-se avaliar se a Escala de Analogia Visual (EAV) de Apetite pode ser útil como ferramenta para prevenção de ganho de peso indesejável ou da redução da perda ponderal esperada em pacientes submetidos à gastroplastia. Trata-se de um estudo longitudinal, onde trinta pacientes submetidos à gastroplastia, selecionados aleatoriamente, tiveram seu apetite aferido no primeiro e terceiro mês após a realização da cirurgia. Na EAV de apetite utilizada constavam dados referentes à sensação subjetiva de fome, saciedade, plenitude e desejo de comer alimentos em geral ou específicos (doce, salgado, temperado e gorduroso). A escala, para cada item, tem o comprimento de $100 \mathrm{~mm}$, com numeração de 0 a 10, onde o próprio paciente assinala uma linha vertical no ponto onde considera estar sua sensação de apetite para o item avaliado. Os dados foram analisados através do teste t de Student, ao nível de 5\%. No primeiro mês, houve estabilidade das sensações referentes a todos os itens, compatível com ausência de vontade de ingerir cada tipo de alimento. No entanto, este fato não ocorreu aos três meses após a cirurgia, havendo menor plenitude e saciedade e maior desejo de comer alimentos específicos. Este fato pode se traduzir em uma maior busca quantitativa por estes alimentos, favorecendo ganho ponderal ou redução da velocidade de perda ponderal. Conclui-se que a EAV de apetite utilizada é uma ferramenta que pode ser útil no acompanhamento deste tipo de paciente, direcionando as estratégias da equipe para uma maior efetividade.

Palavras-chave: Fome; Escala de analogia visual de apetite; Obesidade; Gastroplastia. 


\begin{abstract}
The aim was to assess whether the appetite Visual Analogy Scale (VAS) can be useful as a tool for preventing unwanted weight gain or reducing the expected weight loss in patients undergoing gastroplasty. This is a longitudinal study, in which thirty patients submitted to gastroplasty, randomly selected, had their appetite measured in the first and third months after the surgery. The appetite VAS used contained data referring to the subjective feeling of hunger, satiety, fullness and desire to eat foods in general or specific (sweet, salty, spicy and greasy). The scale, for each item, has a length of $100 \mathrm{~mm}$, with a number from 0 to 10 , where the patient himself marks a vertical line at the point where he feels his feeling of appetite for the item evaluated. The data were analyzed using the Student's t test, at the level of $5 \%$. In the first month, there was stability in the sensations related to all items, compatible with the lack of desire to eat each type of food. However, this fact did not occur at three months after surgery, with less fullness and satiety and greater desire to eat specific foods. This fact can translate into a greater quantitative search for these foods, favoring weight gain or reducing the speed of weight loss. It is concluded that the appetite VAS used is a tool that can be useful in monitoring this type of patient, directing the team's strategies for greater effectiveness.
\end{abstract}

Keywords: Hunger; Appetite visual analogy scale; Obesity; Gastroplasty.

\title{
Resumen
}

El objetivo fue avaluar si la Escala de Analogía Visual del Apetito (EAV) puede ser útil como herramienta para prevenir el aumento de peso no deseado o reducir la pérdida de peso esperada en pacientes sometidos a gastroplastia. Se trata de un estudio longitudinal, en el que se midió el apetito en treinta pacientes sometidos a gastroplastia, seleccionados aleatoriamente, en el primer y tercer mes después de la cirugía. La EAV del apetito utilizada contenía datos referentes a la sensación subjetiva de hambre, saciedad, plenitud y ganas de ingerir alimentos en general o específicos (dulces, salados, picantes y grasos). La escala, para cada ítem, tiene una longitud de $100 \mathrm{~mm}$, con un número del 0 al 10, donde el propio paciente marca una línea vertical en el punto donde siente su sensación de apetito por el ítem evaluado. Los datos se analizaron mediante la prueba t de Student, al nivel del 5\%. En el primer mes, hubo estabilidad en las sensaciones relacionadas con todos los ítems, compatible con la falta de ganas de ingerir cada tipo de alimento. Sin embargo, este hecho no ocurrió a los tres meses de la cirugía, con menor plenitud y saciedad y mayor deseo de ingerir alimentos específicos. Este hecho puede traducirse en una mayor búsqueda cuantitativa de estos alimentos, favoreciendo el aumento de peso o reduciendo la velocidad de la pérdida de peso. Se concluye que la EAV del apetito utilizada es una herramienta que puede ser útil en el seguimiento de este tipo de pacientes, orientando las estrategias del equipo para una mayor efectividad.

Palabras clave: Hambre; Escala de analogía visual del apetito; Obesidad; Gastroplastia.

\section{Introdução}

O tratamento para obesidade, a partir da cirurgia bariátrica, é uma alternativa importante e eficaz em casos de indivíduos com índice de Massa Corporal (IMC) maior que $40 \mathrm{~kg} / \mathrm{m}^{2}$, ou $35 \mathrm{~kg} / \mathrm{m}^{2}$ com uma ou mais comorbidades graves relacionadas, que não obtiveram sucesso a partir de outros tipos de tratamento, como medicamentoso e relacionado a mudanças no estilo de vida. Assim, o tratamento cirúrgico pode proporcionar saciedade precoce e melhora de comorbidades clínicas (ABESO, 2016).

A educação em saúde tem papel fundamental nas ações relacionadas à obesidade e representa um desafio para os profissionais, os quais devem estar bem preparados (Heine et al., 2021). As ações educativas no pós-operatório de cirurgia bariátrica são essenciais para reduzir a probabilidade de complicações e devem se desenvolver com a participação ativa do indivíduo, valorizando as constantes atualizações relacionadas ao paciente (Araújo et al., 2018).

O ensino na saúde realizado à luz da andragogia é capaz de desenvolver nos adultos toda a sua capacidade, permitindo que suas habilidades sejam devidamente afloradas, assim como a continuidade da utilização de métodos. A aprendizagem pela andragogia é voltada para a resolução de problemas que se confrontam permanentemente com o cotidiano, o que desvincula uma lógica conteudista, havendo a compreensão da utilidade prática do conteúdo em questão (Oliveira et al., 2019).

Em condições relacionadas ao processo de emagrecimento de sujeitos que se submeteram à cirurgia bariátrica, ou gastroplastia, considera-se a forte influência de conceitos e práticas andragógicas inseridas na significação, na resignificação e na aplicação de conceitos trabalhados ao longo do tratamento de obesidade (Rodrigues, 2017). 
Além das comorbidades relacionadas ao adoecimento físico, o indivíduo com obesidade, na maioria das vezes, apresenta um estado de sofrimento psíquico observado pela condição de desajustamento emocional (depressão, ansiedade, alterações de humor, etc.) e formação de um autoconceito negativo (vergonha, insegurança, autodepreciação, baixa autoestima, etc.) acarretados pela discriminação imposta pela sociedade frente ao estigma da obesidade, o qual determina os indivíduos com obesidade como pessoas preguiçosas e irresponsáveis (Fulton et al., 2021).

Os problemas psicológicos do indivíduo com obesidade podem persistir após o procedimento de cirurgia bariátrica, bem como interferir na significação da aprendizagem trabalhada por profissionais da saúde, uma vez que o caminho andragógico é influenciado pelas experiências individuais do sujeito (Van Zyl et al., 2020).

A utilização da Escala de Analogia Visual (EAV) de apetite pode auxiliar os profissionais e os pacientes na identificação de barreiras à adequada perda ou manutenção ponderal. Sensações subjetivas de apetite não se relacionam apenas com o estar ou não com o estômago vazio, mas com todas as experiências e emoções prévias do indivíduo, as quais podem se refletir no desejo de comer em geral ou de ingerir um alimento em particular. Tal escala não foi ainda utilizada com o intuito de identificar se o tempo decorrido da realização da gastroplastia pode levar a sensações diferenciadas no que tange ao desejo de comer. Pode-se hipotetizar que, caso haja aumento no desejo de comer, tal fato pode se constituir em entrave à efetividade das ações educativas junto a este tipo de paciente, a menos que as estratégias sejam direcionadas a estes novos fatos.

Portanto, o objetivo do presente estudo foi avaliar se esta ferramenta pode ser útil na prevenção de ganho de peso indesejável ou redução da perda ponderal esperada em pacientes submetidos à gastroplastia.

\section{Metodologia}

Tratou-se de estudo prospectivo, quantitativo com abordagem descritiva e analítica realizado no Núcleo do Obeso do Ceará (NOC), um centro de referência de tratamento da obesidade situado na cidade de Fortaleza, Ceará, no período de setembro a novembro de 2014.

Estudos quantitativos englobam a coleta de dados quantitativos ou numéricos, os quais geram conjuntos de dados que podem ser analisados por meio de técnicas matemáticas como é o caso da análise estatística. Estudos na área de Saúde podem utilizar métodos estatísticos para realizar previsões em relação a algum fenômeno em estudo. Para um tratamento estatístico com mais rigor, torna-se necessária a definição do espaço amostral, que é o universo de realização da pesquisa ou o conjunto no qual se vai levantar dados, além da definição da amostra, ou seja, quantidade de indivíduos suficiente para ser considerado como um estudo estatístico válido (Pereira et al., 2018).

A amostra foi obtida por conveniência, selecionando-se aleatoriamente 30 indivíduos que estavam em tratamento após a cirurgia bariátrica. Os critérios de inclusão foram: faixa etária de 18 a 60 anos, ter realizado a gastroplastia e estar em atendimento no local citado. Foram excluídos os pacientes desistentes do tratamento bariátrico, bem como os que não desejaram continuar a participar da investigação.

Para a coleta de dados, aplicou-se um instrumento estruturado, junto ao paciente, incluindo dados demográficos, socioeconômicos, antecedentes relacionados com a obesidade e dados antropométricos. No instrumento foi também exibida a EAV de apetite adaptada da escala construída por Flint et al. (2000), para preenchimento pelo próprio paciente. A entrevista ocorreu em dois momentos: no primeiro e no terceiro mês de pós-operatório de gastroplastia, sendo que no segundo momento foram aferidos novamente apenas os dados antropométricos e relativos à EAV de apetite. Esta escala, já utilizada no Brasil, é composta por 8 itens sobre os sentimentos atuais de fome, saciedade, plenitude e desejo de comer alimentos, em geral ou específicos (doce, salgado, temperado e gorduroso) (Nobre et al., 2006; Lins et al., 2010). 
Foram exibidos, em cada item de todas as questões, valores extremos de sensação (presente ou ausente) indicados em uma linha de $100 \mathrm{~mm}$ de comprimento, sem graduação. Os voluntários foram orientados a marcar uma linha vertical no ponto da escala em que achassem estar a sua sensação referente ao item investigado. O ponto marcado pelo indivíduo foi medido com o auxílio de uma régua, da extremidade esquerda (pontuação mínima zero mm) para a direita (pontuação máxima 100 $\mathrm{mm}$ ), a fim de quantificar as medidas resultantes (Lins et al., 2010; Lluch et al., 2010).

Os procedimentos seguiram as recomendações de Flint et al. (2000) e Lluch et al. (2010). O preenchimento da escala se deu, nas duas ocasiões, nos mesmos horários, entre 16 horas e 18 horas.

Todo o preenchimento das escalas foi acompanhado pelo pesquisador, a fim de elucidar possíveis dúvidas e auxiliar nas dificuldades, sem interferir nos resultados indicados pelos pacientes.

Os dados antropométricos (peso e altura) foram coletados do prontuário, antes da realização da gastroplastia, sendo o peso aferido novamente no primeiro e terceiro contato. Com estes dados foram calculados os índices de massa corporal (IMC) médios prévio à cirurgia, no primeiro e no terceiro mês após a mesma, categorizando o excesso ponderal de acordo com a World Health Organization (1998).

Utilizou-se o Excel for Windows, versão 14.0, (Microsoft) para armazenar os dados. A análise estatística foi realizada através do Teste $t$ de Student, utilizando-se o Statistical Package for the Social Sciences (SPSS) v. 18.0 (Motta et al., 2009).

O estudo foi previamente aprovado pelo Comitê de Ética em Pesquisa com Seres Humanos da Universidade Estadual do Ceará, sob o número 770.862 e todos os pacientes assinaram o termo de consentimento livre e esclarecido para a participação no mesmo.

\section{Resultados}

Considerando-se o perfil demográfico e socioeconômico do grupo entrevistado, constatou-se uma maioria do sexo feminino (70,0\%), cor autorreferida branca $(90,0 \%)$, na faixa etária de 30 a 59 anos $(60,0 \%)$, com cônjuge (56,7\%), com mais de 8 anos de estudo (80,0\%), ocupação remunerada $(53,4 \%)$ e recebendo mais de 2 salários mínimos mensais $(60,0 \%)$. Em relação aos antecedentes relacionados à obesidade, verificou-se que $80 \%$ dos pacientes apresentavam histórico familiar de obesidade, 33,3\% relataram início da obesidade ainda na infância e 50\% citaram tempo de obesidade maior ou igual a 20 anos.

Acompanhou-se a variação do IMC da população analisada e constatou-se que houve uma redução gradativa do excesso ponderal ao longo do tempo avaliado, com ausência de obesidade mórbida aos 3 meses após a cirurgia, como exposto na Tabela 1. 
Tabela 1. Distribuição percentual dos pacientes do Núcleo do Obeso do Ceará avaliados $(\mathrm{n}=30)$, segundo período de mensuração e categorias de excesso de peso. Fortaleza, 2014.

\begin{tabular}{ccc} 
Período da Mensuração & Categoria de excesso & Percentual (\%) \\
\hline \multirow{2}{*}{ Antes da cirurgia } & Obesidade Mórbida $(\geq 40)$ & 76,7 \\
& Obesidade grau II $(35-39,9)$ & 23,3 \\
& Obesidade grau I $(30-34,9)$ & - \\
& Sobrepeso $(25,0-29,9)$ & - \\
Primeiro mês pós-opreratório & Obesidade Mórbida $(\geq 40)$ & 26,7 \\
& Obesidade grau I $(30-34,9)$ & 53,3 \\
& Sobrepeso $(25,0-29,9)$ & 18,1 \\
\hline Terceiro mês pós-operatótio & Obesidade Mórbida $(\geq 40)$ & 1,9 \\
& Obeso II $(35-39,9)$ & 21,2 \\
& Obeso I (30-34,9) & 65,5 \\
\hline
\end{tabular}

${ }^{1}$ Segundo IMC $\left(\mathrm{Kg} / \mathrm{m}^{2}\right)$, categorizado de acordo com World Health Organization (1998).

Fonte: Elaborado pelos autores.

O Gráfico 1 exibe os achados comparativos, ao longo do tempo, referentes à EAV de apetite. Observa-se que há diferença estatística nas médias encontradas para a maioria das questões avaliadas, mesmo com os valores ainda se mantendo longe do desejo extremo de ingestão alimentar.

Considerando a questão 1, relativa à sensação de fome, observa-se que, mesmo em ambos os períodos os valores terem sido baixos (quanto mais baixo, menor a percepção de forme) e mesmo parecendo haver uma elevação desta sensação aos 3 meses pós-cirurgia, a diferença não foi significante $(\mathrm{p}=0,999)$.

A questão 2 refere-se à saciedade, sendo que quanto mais elevados forem os valores, maior a saciedade. Ocorreu uma redução dos valores aos 3 meses, evidenciando menor percepção de saciedade $(\mathrm{p}<0,001)$.

Da mesma forma, aos 3 meses a plenitude é menos sentida $(\mathrm{p}<0,001)$ do que no primeiro mês (questão 3).

Da mesma forma que ocorreu para a questão 1, o desejo de comer (questão 4) parece maior aos 3 meses, mas sem diferença estatística $(\mathrm{p}=1,000)$.

As questões 5, 6, 7 e 8 referem-se ao desejo por alimentos específicos, onde quanto mais altos os valores menor o desejo demonstrado pelos mesmos. Observa-se mais uma vez que há uma redução das médias em todas as quatro situações indagadas, respectivamente doce $(\mathrm{p}=0,001)$, salgado $(\mathrm{p}<0,001)$, temperado $(\mathrm{p}<0,001)$ e gorduroso $(\mathrm{p}=0,033)$. 
Gráfico 1. Médias dos resultados da escala de analogia visual de apetite aplicada com os pacientes do Núcleo do Obeso do Ceará avaliados no primeiro e no terceiro mês pós gastroplastia. *Diferença significante (p<0,05). Fortaleza, 2014.

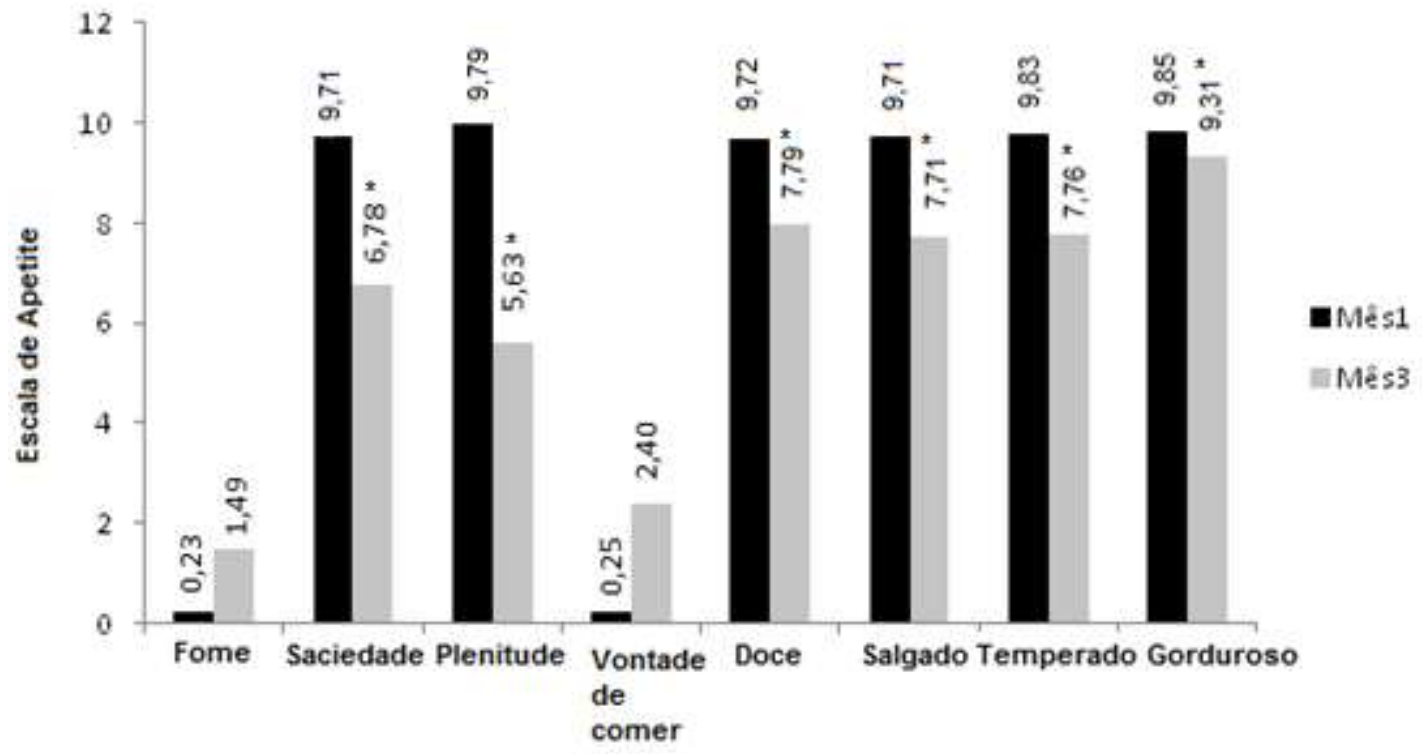

Fonte: Elaborado pelos autores.

\section{Discussão}

Embora se trate de um estudo com amostra de conveniência, vale comentar alguns aspectos do perfil demográfico e socioeconômico do grupo avaliado em comparação com outros estudos que também envolveram pacientes candidatos ou submetidos à cirurgia bariátrica. Assim, o predomínio do sexo feminino, um bom nível de instrução e uma média de renda superior a dois salários mínimos também foram constatados em outro estudo que caracterizou pacientes com obesidade grave atendidos em Centros de Referência em Obesidade da cidade do Rio de Janeiro (Reis, 2018). Perfil semelhante também foi encontrado em investigação com indivíduos pernambucanos (Nóbrega et al., 2020) e ingleses (Erdogdu et al., 2019) submetidos à cirurgia bariátrica.

Os dados observados neste estudo revelam, ainda, que a obesidade é uma questão inserida no contexto familiar e histórico dos participantes, dada a alta proporção de relatos de antecedentes familiares de obesidade e de tempo prolongado estando com obesidade. Tal fato também é relatado por outras análises (Cafure et al., 2018; Lisowski et al., 2019).

Observou-se, a partir dos presentes dados, a ausência de obesidade mórbida na amostra no $3^{\circ}$ mês após a cirurgia, sendo que a maioria destes pacientes estava redistribuída em obesidade grau 2, obesidade grau 1 e sobrepeso já após um mês do procedimento. Esta redistribuição ficou ainda melhor aos 3 meses, demonstrando a redução gradativa do IMC, a qual também é observada em outras pesquisas.

Em análise realizada com pacientes submetidos à cirurgia bariátrica do estado do Mato Grosso, foi observado IMC médio pré-cirúrgico de $50 \mathrm{~kg} / \mathrm{m}^{2}$, enquanto, após um ano da cirurgia, este passou a 34,1 kg/ m² e, após 2 anos, a $32,7 \mathrm{~kg} / \mathrm{m}^{2}$ (Kato et al., 2018). Em estudo paranaense, o IMC médio dos pacientes passou de $47 \mathrm{~kg} / \mathrm{m}^{2}$ para $32,3 \mathrm{~kg} / \mathrm{m}^{2} \mathrm{dentro}$ de um período pós-cirúrgico médio de 4 anos (Rossetti et al., 2017).

No caso de pesquisa pernambucana, após realização da cirurgia, a maioria dos pacientes, que tinham obesidade mórbida, passaram a ter obesidade grau II após 1 ano de acompanhamento (Silva et al., 2017), situação esta que aconteceu neste presente estudo apenas 1 mês após o procedimento. Dessa forma, a obesidade mórbida também se tornou ausente, apesar 
de ter ocorrido num período de tempo superior ao do presente estudo. Na realidade, o grupo estudado nesta pesquisa, nas fases de avaliação, apresentou evolução além do esperado.

Pode-se observar que a redução do IMC decorrente da cirurgia bariátrica é bastante variável, sendo esperada uma perda de 60 a 75\% do excesso de peso corporal, no período de 18 a 24 meses após o procedimento (Bradley et al., 2017). O tratamento cirúrgico é considerado bem sucedido se houver perda de, no mínimo, 50\% do peso excedente referido no momento da operação e se o indivíduo apresentar IMC fora da classificação de obesidade grau III. Alguns autores estabelecem classificação para sucesso de pós-operatório de acordo com os resultados sobre o IMC. O resultado é considerado excelente quando o IMC fica menor que $30 \mathrm{~kg} / \mathrm{m}^{2}$, caracterizando ausência de obesidade, e bom quando fica entre $30-35 \mathrm{~kg} / \mathrm{m}^{2}$ por volta do final do primeiro ano após a cirurgia (Bastos et al., 2013).

Segundo a Associação Brasileira para o Estudo da Obesidade e da Síndrome Metabólica (ABESO, 2016), além de atingir o parâmetro de sucesso cirúrgico, é necessária a manutenção da perda de peso por cinco anos. Por outro lado, estudo de revisão apontou que pacientes acompanhados a curto prazo (até 2 anos) refletiram ótimo resultado para redução de excesso de peso, enquanto pesquisas de médio a longo prazo (mais que 4 anos) demonstram que, embora ainda haja perda de peso, pode ter início o reganho de peso (Felsenreich et al., 2019). Também já foi relatado que a diminuição acentuada do peso e da gordura corporal poderia ocorrer até o $12^{\circ}$ (Kops, 2020) ou $18^{\circ}$ (Magalhães, 2020) mês pós-operatório, momento em que inicia a desaceleração e possível reganho. Seguindo essa linha, no estudo Mato-Grossense supracitado (Kato et al., 2018), houve uma desaceleração da perda de peso com o passar do tempo.

Tem-se mais uma razão para se monitorar de perto estes pacientes, a fim de que bons resultados, como estes relatados pela presente amostra no primeiro trimestre, evoluam a contento ao longo dos anos seguintes. Uma das ferramentas de monitorização, portanto, pode ser a EAV de apetite.

A aplicação da EAV de apetite evidenciou que após 3 meses decorridos da cirurgia, a relação do paciente com a alimentação se modifica. As sensações de fome e a vontade de comer em geral não apresentaram diferença estatística do primeiro para o terceiro mês, podendo estas estarem sendo afetadas pelo tamanho pequeno da amostra ou pelo tempo de observação da evolução, de apenas 3 meses. Entretanto, a sensação de fome foi baixa tanto aos 30 dias como aos 3 meses após a cirurgia. Por outro lado, a saciedade e plenitude se encontraram reduzidas, ao mesmo tempo em que se elevou o desejo por alimentos doces, salgados, temperados e gordurosos após 3 meses, ou seja, alimentos que, a depender da quantidade, podem levar ao ganho de peso.

Utilizando-se escalas de analogia visual, outros estudos também demonstraram a redução na sensação de fome em curto período pós-cirúrgico, como de 2 semanas a 1 mês. Entretanto, esta sensação retornou aos níveis pré-cirúrgicos após 3 meses do procedimento (Sanmiguel et al., 2017; Smith et al., 2020). Esta sensação de fome parece sofrer mudanças rápidas, além de não ter sido encontrada diferença entre $1^{\circ}$ e $3^{\circ}$ mês pós-cirúrgico, revisão sistemática também evidenciou que esta permaneceu inalterada após 6 meses de cirurgia. Já em relação à saciedade e plenitude pós-prandial, estas foram verificadas como mais elevadas além de 6 meses após a cirurgia (Redpath et al., 2021).

São descritos alguns fatores que podem levar a redução da sensação de fome e consequente perda de peso após a cirurgia bariátrica. Estes fatores podem ser a redução da capacidade gástrica, bem como fatores hormonais, como diminuição da grelina, diminuição da resistência à leptina e aumentos de peptídeo YY e o glucagon-like peptideo-1 (GLP-1), melhorando o controle da fome e da saciedade (Silva et al., 2013).

Em relação aos alimentos doces e gordurosos, alguns estudos relataram redução no desejo por estes alimentos nos períodos de 2 semanas (Smith et al., 2020), 1 mês (Van Duinkerken et al., 2021) e 2 anos (Redpath et al., 2021) após a cirurgia. Entretanto, além do presente estudo ter relatado aumento no desejo por esses alimentos aos 3 meses pós-cirúrgicos, também foi 
descrito que esse desejo pode aumentar gradualmente até 3 anos depois do procedimento, podendo se tornar uma ingestão diária (Magalhães, 2020).

Esta realidade deve funcionar como um alerta para a equipe que atende estes pacientes, pois a situação evidencia uma adaptação e o retorno do consumo em excesso. A partir daí, torna-se necessário investigar se as sensações estão já começando a se traduzir em comportamentos e se, na presença de desejos alimentares, estes estão sendo satisfeitos em quantidades controladas ou se o paciente já começa a perceber sinais de descontrole de ingestão.

A partir da concretização de mudança de vida, evidenciada com a perda ponderal progressiva, a falta de acompanhamento profissional pode levar a maior reganho de peso (Gil-Rendo et al., 2019). Em consonância, pacientes não acompanhados são mais propensos a interromper o controle diário de ingestão alimentar, reduzindo seu automonitoramento (Yu et al., 2019).

Para que esta situação seja amenizada, faz-se necessária a busca, por parte da equipe interdisciplinar aliada à família do paciente, de recursos andragógicos capazes de impactarem sobre as questões de significação e ressignificação das sensações alimentares, uma vez que elas podem interferir de fato nos aspectos comportamentais do indivíduo com obesidade em tratamento bariátrico. Assim, o acompanhamento da equipe é imprescindível para o sucesso do tratamento, principalmente nos primeiros meses após a cirurgia, os quais são fundamentais para a disposição e o manejo dos novos hábitos que deverão ser seguidos por toda a vida (Bauer et al., 2017).

A aplicação da EAV de apetite possibilita à equipe quantificar as sensações alimentares e analisar os aspectos que merecem atenção mais imediata para o desenvolvimento de ações educativas mais efetivas. A equipe realmente pode utilizar os relatos das sensações alimentares como diretrizes para delineamento de estratégias em ensino na saúde.

Constatou-se, entretanto, através de busca bibliográfica, a ausência de outros estudos que mostrem a aplicação de EAV no contexto da gastroplastia com a proposta aqui aplicada. Há publicações que utilizam a escala em resposta a refeições padronizadas, a fim de medir níveis de peptídeos gastrointestinais envolvidos nos mecanismos de regulação de fome e saciedade ou em avaliação de respostas a refeições segundo concentração maior ou menor de calorias (Andriessen et al., 2018; Torquati et al., 2019), mas não com uma proposta de aplicação prática para aprimorar o atendimento deste tipo de paciente, assegurando sua melhor resposta terapêutica.

Há, portanto, necessidade de maiores investigações acerca da quantificação das modificações das sensações alimentares como elemento norteador de estratégias de abordagem ao paciente bariátrico.

O estudo apresenta algumas limitações. Uma delas é o tamanho pequeno da amostra e outra é o tempo de acompanhamento de apenas 3 meses, já comentados, o que pode ter dificultado encontrar algumas associações. Por outro lado, mesmo com tais limitações foi possível perceber mudanças na maioria das questões integrantes da escala aplicada. Ressalte-se, ainda, que a escala não foi aplicada imediatamente antes ou após as refeições, como costuma ser em pesquisas, mas a proposta aqui foi avaliar sua aplicabilidade em contexto real de atendimento e aí, sim, padronizou-se a verificação sempre no mesmo horário, para eliminar este possível viés.

\section{Conclusão}

A Escala de Apetite forneceu subsídios para análise das sensações alimentares dos pacientes, mostrando-se um instrumento avaliativo de fácil aplicação e entendimento. A partir dela, a equipe interdisciplinar pode analisar as variantes entre a fome e a saciedade em curto, médio e longo prazo. Assim, a EAV de apetite pode ser ferramenta de trabalho importante para harmonizar as estratégias de Ensino na Saúde com as percepções dos pacientes, evitando-se o reganho de peso ponderal ou a redução da perda desejada. 
Além disso, este estudo pode servir de base para investigações futuras, a serem desenvolvidas a partir de estudos longitudinais, com acompanhamento a longo prazo de indivíduos que tenham sido submetidos à cirurgia bariátrica. Ademais, também incita estudos de cunho experimental, com intervenções alimentares que utilizem a escala para avaliar as sensações subjetivas de apetite antes e após ingestão de refeições com composições específicas.

\section{Referências}

Andriessen, C., Christensen, P., Nielsen, L. V., Ritz, C., Astrup, A., Larsen, T. M., Martinez, A., Saris, W. H. M., Van Baak, M. A., Papadaki, A., Kunesova, M., Jebb, S., Blundell, J., Lawton, C., \& Raben, A. (2018). Weight loss decreases self-reported appetite and alters food preferences in overweight and obese adults: Observational data from the DiOGenes study. Appetite, 125, 314-322. https://doi.org/10.1016/j.appet.2018.02.016

Araújo, B. N., Galina, D., Cantele, A. B., Lopes, S., Ramos, A. I., \& Trentin, P. (2018). Cirurgia Bariátrica em adultos: a importância da orientação multiprofissional no período pós-operatório. Revista uningá, 55(4), 187-199.

Associação Brasileira para o Estudo da Obesidade e da Síndrome Metabólica (ABESO) (2016). Diretrizes brasileiras de obesidade 2016 (4 ed).

Bastos, E. C. L., Barbosa, E. M. W. G., Soriano, G. M. S., Santos, E. A. D., \& Vasconcelos, S. M. L. (2013). Fatores determinantes do reganho ponderal no pós-operatório de cirurgia bariátrica. ABCD. Arquivos Brasileiros de Cirurgia Digestiva (São Paulo), 26, 26-32. https://doi.org/10.1590/S010267202013000600007

Bauer, E., Giovanardi, H. J., Alves, M. K., \& de Souza, J. S. M. (2017). Complexidade da obesidade antes e após a cirurgia bariátrica método sleeve. RBONERevista Brasileira De Obesidade, Nutrição E Emagrecimento, 11(68), 653-660.

Bradley, L. E., Forman, E. M., Kerrigan, S. G., Goldstein, S. P., Butryn, M. L., Thomas, J. G., Herbert, J. D. \& Sarwer, D. B. (2017). Project HELP: a remotely delivered behavioral intervention for weight regain after bariatric surgery. Obesity surgery, 27(3), 586-598. https://doi.org/10.1007/s11695-0162337-3

Cafure, F., Schmidt, J., Duré, L. S., Furbeta, P. H., Moraes, R., Arruda, R., \& Gaban, S. (2018). Prevalência de excesso de peso e obesidade central em acadêmicos do curso de Medicina da Universidade UNIDERP. RBONE-Revista Brasileira De Obesidade, Nutrição E Emagrecimento, 12(69), 94-100.

Erdogdu, U. E., Cayci, H. M., Tardu, A., Demirci, H., Kisakol, G., \& Guclu, M. (2019). Health literacy and weight loss after bariatric surgery. Obesity surgery, 29(12), 3948-3953. https://doi.org/10.1007/s11695-019-04060-7

Felsenreich, D. M., Langer, F. B., \& Prager, G. (2019). Weight loss and resolution of comorbidities after sleeve gastrectomy: a review of long-term results. Scandinavian Journal of Surgery, 108(1), 3-9. https://doi.org/10.1177/1457496918798192

Flint, S. J., Enquist, L. W., Krug, R. M.; Racaniello, V. R.; Skalka, A. M. (2000). Principles of virology: molecular biology, pathogenesis and control.

Fulton, M., \& Srinivasan, V. N. (2021). Obesity, Stigma And Discrimination.

Gil-Rendo, A., Muñoz-Rodríguez, J. R., Bardají, F. D., Trujillo, B. M., Martínez-de Paz, F., González, M. D. P. C., Medina, I. A., \& Fernández, J. M. (2019). Laparoscopic sleeve gastrectomy for high-risk patients in a monocentric series: long-term outcomes and predictors of success. Obesity surgery, 29(11), 36293637. https://doi.org/10.1007/s11695-019-04044-7

Heine, M., Lategan, F., Erasmus, M., Lombaard, C. M., Mc Carthy, N., Olivier, J., Van Niekerk, M. \& Hanekom, S. (2021). Health education interventions to promote health literacy in adults with selected non-communicable diseases living in low-to-middle income countries: A systematic review and meta-analysis. Journal of Evaluation in Clinical Practice, 22. https://doi.org/10.1111/jep.13554.

Kato, K. R. A., Brim, C. A., Pizarro, C. B., de Alencar Miranda, A., Yonamine, F., \& Carvalho, P. M. (2018). Análise de variação de IMC de pacientes submetidos à cirurgia bariátrica. COORTE-Revista Científica do Hospital Santa Rosa, (08).

Kops, N. L. (2020). Fatores associados à perda de peso após cirurgia bariátrica. Tese (Doutorado - Programa de Pós-Graduação de Ciências Médicas: Endocrinologia) - Universidade Federal do Rio Grande do Sul.

Lins, T. A., Neves, P. R. D. S., Costa, M. D. C., \& Prado, W. L. D. (2010). Efeitos de diferentes intensidades de exercício sobre o gasto energético e a sensação de fome em jovens. Revista Brasileira de Cineantropometria \& Desempenho Humano, 12(5), 359-366. https://doi.org/10.5007/1980$0037.2010 v 12$ n5p359

Lisowski, J. F., Leite, H. M., Bairros, F., Henn, R. L., Costa, J. S. D. D., \& Olinto, M. T. A. (2019). Prevalência de sobrepeso e obesidade e fatores associados em mulheres de São Leopoldo, Rio Grande do Sul: um estudo de base populacional. Cadernos Saúde Coletiva, 27(4), 380-389. https://doi.org/10.1590/1414462X201900040226

Lluch, A., Hanet-Geisen, N., Salah, S., Salas-Salvadó, J., L’Heureux-Bouron, D., \& Halford, J. C. (2010). Short-term appetite-reducing effects of a low-fat dairy product enriched with protein and fibre. Food quality and preference, 21(4), 402-409. https://doi.org/10.1016/j.foodqual.2009.10.001

Magalhães, B. R. (2020). Determinantes do sucesso da cirurgia bariátrica.

Motta, V. T., \& Oliveira Filho, P. F. (2009). SPSS: Análise de dados biomédicos.

Nobre, L. N., Bressan, J., Costa Sobrinho, P. D. S., Costa, N. M. B., Minin, V. P. R., \& Cecon, P. R. (2006). Volume de iogurte light e sensações subjetivas do apetite de homens eutróficos e com excesso de peso. Revista de Nutrição, 19(5), 591-600. https://doi.org/10.1590/S1415-52732006000500007 
Nóbrega, M. P., Cabral, P. C., Pinho, C. P. S., Costa, J., \& de Lima, D. S. C. (2020). Perfil alimentar e reganho de peso de pacientes submetidos à cirurgia bariátrica em um hospital universitário. Brazilian Journal of Development, 6(12), 94771-94785. https://doi.org/ 10.34117/bjdv6n12-094

Oliveira, B., Paixão, I. C., Inhoato, K. C., \& Fonseca, J. R. (2019). Contribuições da neurociência e da neuropsicologia ao processo de aprendizagem adultaandragogia. Revista Diálogos Interdisciplinares, 1(7), 93-107.

Pereira, A. S., Shitsuka, D. M., Parreira, F. B., \& Shitsuka, R. (2018). Metodologia da pesquisa científica.

Redpath, T. L., Livingstone, M. B. E., Dunne, A. A., Boyd, A., le Roux, C. W., Spector, A. C., \& Price, R. K. (2021). Methodological issues in assessing change in dietary intake and appetite following gastric bypass surgery: A systematic review. Obesity Reviews. https://doi.org/10.1111/obr.13202

Reis, E. C. (2018). Avaliação do componente ambulatorial especializado da linha de cuidado para obesidade grave na Cidade do Rio de Janeiro. Tese (Doutorado - Programa de Pós-graduação em Saúde Pública) - Fundação Oswaldo Cruz, Escola Nacional de Saúde Pública Sergio Arouca.

Rodrigues, G. B. O. (2017). O viver e o aprender do obeso bariátrico.

Rossetti, F. X., Lorenzi, M. N., \& Gomes, K. S. A. G. (2017). Aspectos nutricionais e qualidade de vida após cirurgia bariátrica em pacientes da atenção primária à saúde. Segurança Alimentar e Nutricional, 24(1), 75-82. https://doi.org/10.20396/san.v24i1.8649757

Sanmiguel, C. P., Jacobs, J., Gupta, A., Ju, T., Stains, J., Coveleskie, K., Lagishetty, V., Balioukova, A., Chen, Y., Dutson, E., Mayer, E., \& Labus, J. (2017) Surgically induced changes in gut microbiome and hedonic eating as related to weight loss: preliminary findings in obese women undergoing bariatric surgery. Psychosomatic medicine, 79(8), 880. https://doi.org/10.1097/PSY.0000000000000494

Silva, J., Monteiro, F. A., Nunes, R. C. M., do Nascimento Costa, J. A. B., \& Tavares, F. C. D. L. P. (2017). Avaliação de aspectos clínicos e nutricionais em obesos em pré e pós-operatório de cirurgia bariátrica em um hospital universitário de João Pessoa-PB. RBONE-Revista Brasileira De Obesidade, Nutrição E Emagrecimento, 11(67), 506-522.

Silva, R. F., \& Kelly, E. O. (2013). Reganho de peso após o segundo ano do Bypass gástrico em Y de Roux. Comunicação em Ciências da Saúde, 24(4), 341350 .

Smith, K. R., Papantoni, A., Veldhuizen, M. G., Kamath, V., Harris, C., Moran, T. H., Carnell, S. \& Steele, K. E. (2020). Taste-related reward is associated with weight loss following bariatric surgery. The Journal of clinical investigation, 130(8). https://doi.org/10.1172/JCI137772

Torquati, A., Shantavasinkul, P. C., Omotosho, P., Corsino, L., \& Spagnoli, A. (2019). Perioperative changes in prouroguanylin hormone response in severely obese subjects after bariatric surgery. Surgery, 166(4), 456-459. https://doi.org/10.1016/j.surg.2019.06.037

Van Duinkerken, E., Bernardes, G., van Bloemendaal, L., Veltman, D. J., Barkhof, F., Mograbi, D. C., Gerdes, V. E. A., Deacon, C. F., Holst, J. J., Drent, M. L., Diamant, M., Kulve, J. T., \& Ijzerman, R. G. (2021). Cerebral effects of glucagon-like peptide-1 receptor blockade before and after Roux-en-Y gastric bypass surgery in obese women: A proof-of-concept resting-state functional MRI study. Diabetes, Obesity and Metabolism, 23(2), 415-424. https://doi.org/10.1111/dom.14233

Van Zyl, N., Andrews, L., Williamson, H., \& Meyrick, J. (2020). The effectiveness of psychosocial interventions to support psychological well-being in postoperative bariatric patients: A systematic review of evidence. Obesity Research \& Clinical Practice. https://doi.org/10.1016/j.orcp.2020.05.005

World Health Organization (WHO). (1998). Obesity Preventing and Managing the Global Epidemic.

Yu, Y., Klem, M. L., Kalarchian, M. A., Ji, M., \& Burke, L. E. (2019). Predictors of weight regain after sleeve gastrectomy: an integrative review. Surgery for Obesity and Related Diseases, 15(6), 995-1005. https://doi.org/10.1016/j.soard.2019.02.009 